\title{
Pure De Novo 4.5 Mb Duplication at Xp11.22-p11.23 in an 18-Month-Old Boy: Phenotypic and Molecular Characterization
}

\author{
Dragan Jovanovic, Nathalie Breland \\ Department of Pathology, Trinity School of Medicine, Alphareta, GA, USA, Saint Vincent and the Grenadines Campus.
}

Correspondence: djovanovic@trinityschoolofmedicine.org; Tel.: + 1-784 456-9751; Fax.: +1-784 456-9715

Received: January 14, 2020; Accepted: February 10, 2020

\begin{abstract}
Objective - The aim of this study was the molecular characterization of a very rare de-novo 4.5 Mb duplication at Xp11.22-p11.23 in an 18-month-old boy with hypotonia and developmental delay, and to correlate these findings with a clinical phenotype and to expand the knowledge of this genetic abnormality. Case Report - The patient is unable to utilize the large muscle systems to move from place to place, assume a stable posture when moving, and to raise himself to a standing position. He is incapable of coherent speech. Single nucleotide polymorphism (SNP) was performed using the Affymetrics Cytoscan High Density platform and maternal fluorescence in situ hybridization (FISH) analysis was used to determine the inheritance pattern. Chromosome microarray test found Xp11.23-p11.22 (48,237,630-52, 737, 268) x2 including numerous OMIM genes (start: SSX4 to end: SSX7) and SHROOM4. Maternal FISH analysis did not detect duplication of Xp11.23. Conclusion - This is de novo change in our patient most likely occurred as a new mutation that was not inherited from either parent.
\end{abstract}

Key Words: X Chromosome • De-Novo • Duplication • Molecular Characterization • Phenotype.

\section{Introduction}

In 2009, Giorda et al. (1) identified inherited and de novo recurrent Xp11.22-p11.23 microduplication in two males and six females. They examined a wide cohort of patients presenting with syndromic intellectual disability (ID). Seventeen new patients with microduplications of Xp11.22-p11.23 were reported in 2015 by Nizon et al. (2). The size of microduplications ranged from 331 kilo base pairs $(\mathrm{Kb})$ to 8.9 mega base pairs $(\mathrm{Mb})$. Patients shared several common major characteristics: language impairment, moderate to severe ID, focal epilepsy with activation during sleep evolving in some patients to continuous spikes-and-waves during slow sleep, abnormal electroencephalogram (EEG), excessive weight, hypotonia, variable and nonspecific anomalies of the extremities and early onset of puberty in females (2). In the most published cases,
$\mathrm{X}$ chromosome microduplication was inherited from the healthy mother. The carrier mothers were asymptomatic because they showed severely skewed $\mathrm{X}$ inactivation of the rearranged $\mathrm{X}$ chromosome. However, it was shown that on the short arm of the $\mathrm{X}$ chromosome, especially on the Xp11 region, clusters of genes can escape inactivation (3) and the excess of $\mathrm{X}$-inactivation escaping genes may be associated with mental impairment (4).

We report here phenotypic and molecular characterization of a de-novo $4.5 \mathrm{Mb}$ duplication at Xp11.23-p11.22 in an 18-month-old boy with hypotonia and developmental delay.

\section{Case Report}

Here we describe an 18-month-old boy (Fig. 1) unable to utilize the large muscle systems in his body 
to move from place to place, assume a stable posture when moving, and to raise himself to a standing position. Floppy since birth, he smiled at 3 months and used a raking grasp. At 12 months he was interactive but the parents' main concern was the child's developmental delays. After receiving physical therapy the boy was making progress. He moved around by rolling but was not speaking. At that period he started to bear weight on his leg and he could sit with support and started waving but was not able to hold his bottle. Self help, raking grasp and cognitive function were delayed. Although he was slightly underweight at birth, his growth at 12 months of age was good. At 18 months the patient was cooperative throughout the evaluation and able to follow one step commands but was not able to extend his arms backward to prevent himself falling. When becoming unbalanced the patient was slow to extend his hands forward or to the side. Also, he was not able to transition to sitting from supine to prone or to pull himself from sitting to standing, and was moving around the environment by rolling. This boy was a second child of a healthy 33-year-old woman and a non-consanguineous 33-year-old man whose family history was unremarkable. Their first child was a 6-year-old healthy girl. The boy was born full-term weighing $3 \mathrm{~kg}$. Pregnancy was complicated by maternal hypertension. He spent 3 extra days in the hospital due to low blood sugar and was treated with an intravenous (IV) sugar drip. His hearing and vision were determined to be functional. Lower lip frenulum was tied and he had fifth finger clinodactyly.

SNP microarray analysis was performed using the Affymetrix Cytoscan HD platform (LabCorp) applying methodology provided by the manufacturer. 250 ng of total extracted genomic deoxyribonucleic acid (DNA) was digested with $\mathrm{NspI}$ and after ligation to NspI adaptors was amplified using Titanium Taq with a GeneAmp polymerase chain reaction (PCR) System 9700. PCR products were purified, DNA was fragmented, biotin labeled and hybridized using Chromosome Analysis Suite. The analysis was based on the GRCh37/hg19 assembly. Truly balanced chromosome alterations will

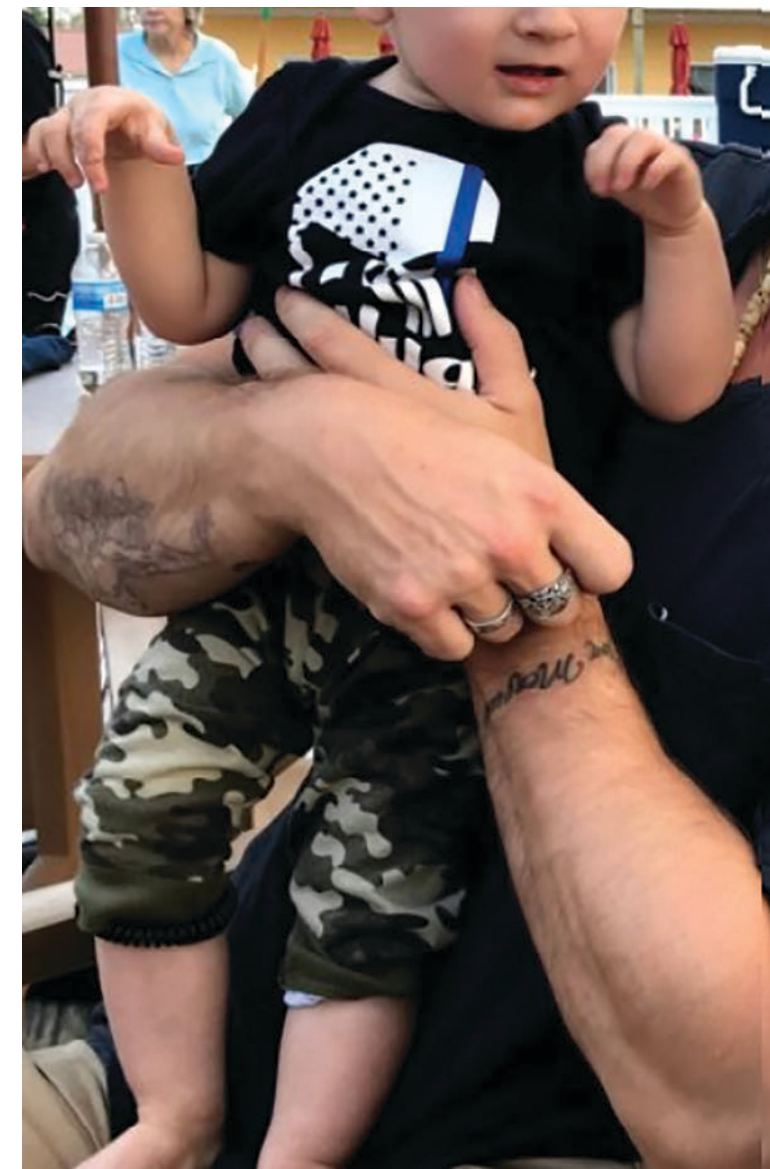

Fig. 1. The Patient Is Unable to Utilize the Large Muscle System to Raise Himself to a Standing Position.

not be detected by this analysis. The threshold for mosaicism is variable, depending on the size of segment. This test was developed and its performance characteristics determined by LabCorp. It has not been cleared or approved by the Food and Drug administration. Multiplanar multisequence magnetic resonance imaging (MRI) brain without and with IV gadolinium was done when the patient was 10 -month-old. Maternal FISH analysis was used to determine inheritance pattern.

Chromosome microarray test found Xp11.22p11.23 (48,237,630-52, 737, 268) x2 including numerous OMIM genes (FTSJ1, SYP, SFROOM4, and PQBP1; start: SSX4 to end: SSX7) and SHROOM4. The hole genome SNP microarray (Reveal) analysis detected an interstitial duplication of chromosomal segment listed (Fig. 2). Ma- 


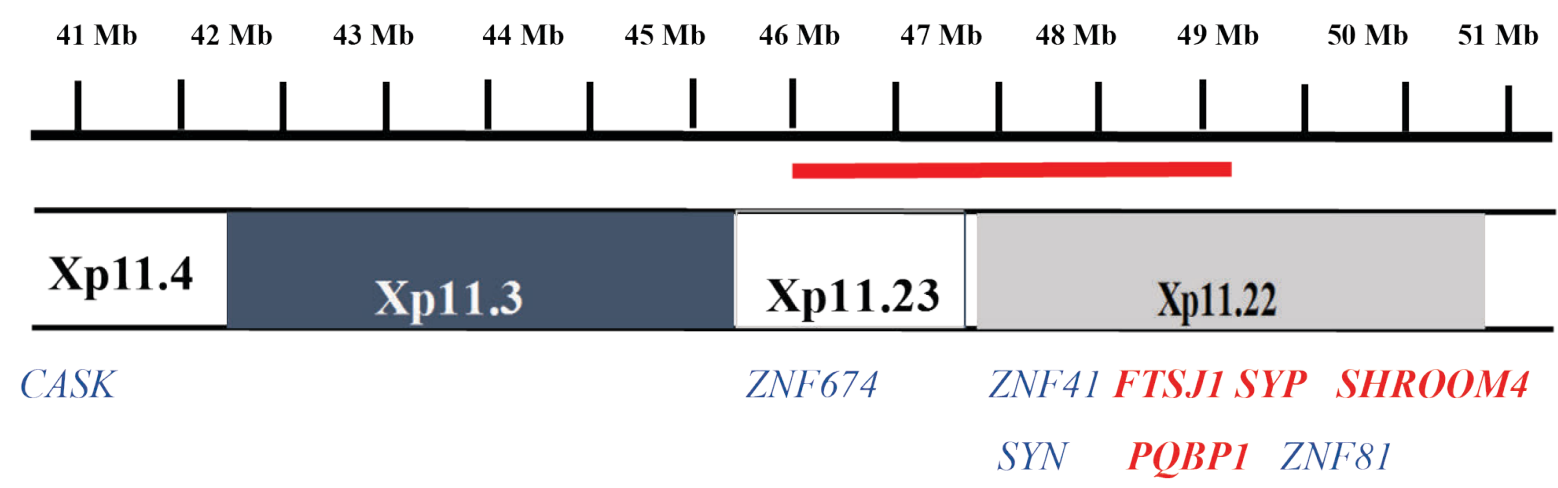

Fig. 2. The Most Common Duplicated Genes in the Xp11 Region. Duplications in Our Patient Are Highlighted in Red.

ternal FISH analysis did not detect duplication of Xp11.23.

\section{Discussion}

Human X chromosome Xp11.22-p11.23 region is susceptible to recurrent chromosome rearrangement resulting in $4.5 \mathrm{Mb}$ microduplication (1). So far it is reported that about 70 people had this duplication. In most cases in women with $\mathrm{X}$ chromosome duplication the $\mathrm{X}$ chromosome with the extra bit is usually inactivated. Since the active X chromosome is the normal one it would not be expected to have any adverse effect on development, health or functioning. For these reasons, the mother of our child patient was advised to perform a genetic analysis with her and based on the results to be given genetic advice. But in some cases inactivation seems to be either random or it is skewed in favor of the X chromosome with the extra bit. In males usually these $\mathrm{X}$ chromosome rearrangements are inherited from a healthy mother.

As in our case the maternal DNA FISH analysis did not find duplication of Xp11.23 we have pure de novo 4.5 Mb duplication. This duplication includes 47 genes of which 14 have already been associated with genetic disorders. Our patient had duplication of POBP1; the mutation was associated with syndromic ID including microcephaly, short stature, spasticity, and occasional heart and renal malformation (5, 6). In 2016, Moey et al. (7) reported that gains in Xp11.22 including IQSEC2 caused ID, were associated with hyperactivity and attention/hyperactivity disorder, and were likely to be dosage-sensitive in males. Protein encoded by $P Q B P 1$ is preferentially expressed in the brain (8). In experimental cellular models overexpression of the POBP1 protein decreased cell growth and increased sensitivity to stress resulting in a loss of Purkinje and glandular cells in the cerebellum and loss of spinal motor neurons (9). In some patients focal seizures were activated during sleep and PQBP1 and SLS35A2 proteins were suggested as candidates for epilepsy (2). Although our patient has no history of epilepsy and EEG was normal, the parents had trouble rousing the boy from sleep on two occasions. He was clammy, disoriented, and have been almost unconscious. Duplicated synaptophysin $(S Y P)$ codes a protein of the synaptic vesicle membrane and mutations have been associated with ID and epilepsy (10). Pinar et al. in 2018 (11) reported a small duplication of only SSX1, SSX9, $S X X 3$ and $S X X 4$ at Xp11.23 in a 23-month-old girl with epilepsy and global developmental delay. The patient had very severe ID and died at 25 months of age from severe pneumonia. Duplicated FTSJ1 and SHROOM4 genes are referred to as candidates for developmental delay (2). SHROOM4 encodes a member of the APX/Shroom family, and encoded protein may play a role in cytoskeletal architecture. The mutation of these genes is found in the Xlinked Stocco dos Santos syndrome characterized by cognitive disabilities $(12,13)$.

It should also be noted that there are people with an Xp11.22 duplication who have developed 
normally and have no speech, behavior, learning or health difficulties (4).

\section{Conclusion}

The patient has a large duplication containing multiple genes many of which are important for normal development. Many children have speech delay, cognitive deficits, sleep disorders and epilepsy. Since the mother does not have the duplication this de novo change in the patient most likely occurred as a new mutation that was not inherited from either parent.

Acknowledgements: We thank the patient, the parents and the physicians for their participation in this study. The parents showed so much love for their child and gave written consent for this study and for the publication of a photo of their son. Institutional Review Board (IRB) functions in our institution also as Ethical Committee. The IRB number for this work is: P-03-2019.

Conflict of Interest: The authors declare that they have no conflict of interest.

\section{References}

1. Giorda R, Bonaglia MC, Beri S, Fichera M, Novara F, Magini $\mathrm{P}$, et al. Complex segmental duplication mediate a recurrent $\operatorname{dup}(\mathrm{X})(\mathrm{p} 11.22$-p11.23) associated with mental retardation, speech delay and EEG anomalies in males and females. Am J Hum Genet. 2009;85(3):394-400. doi: 10.1016/j.ajhg.2009.08.001. Epub 2009 Aug 27.

2. Nizon M, Andrieux J, Rooryck C, de Blois MC, BourelPonchel E, Bourgois B, et al. Phenotype-genotype correlation in 17 new patients with an Xp11.23p11.22 microduplification and review of the literature. Am J Med Genet. 2015;67A(1):111-22. doi: 10.1002/ajmg.a.36807. Epub 2014 Nov 25.

3. Carrel L, Willard HF. X-inactivation profile reveals extensive variability in X-linked gene expression in females. Nature. 2005;434(7031):400-4. doi:10.1038/nature03479.

4. Zhang Y, Castillo-Morales A, Jiang M, Zhu Y, Hu L, Urrutia $\mathrm{AO}$, et al. Genes that escape $\mathrm{X}$-inactivation in humans have high intra-specific variability in expression, are associated with mental impairment but are not slow evolv- ing. Mol Biol Evol. 2013;30(12):2588-601. doi:10.1093/ molbev/mst 148 .

5. Musante L, Kunde SA, Sulistio TO, Fischer U, Grimme A, Frints SG, et al. Common pathological mutation exclusion in PQBP1 induce nonsense-mediated mRNA decay and enhance exclusion of the mutant exon. Hum Mutant. 2010;31(1):90-8. doi:10.1002/humu.21146.

6. Lenski C, Abidi F, Meindl A, Fischer U, Grimme A, Frints $\mathrm{SG}$, et al. Novel truncating mutations in the polyglutamine tract binding protein 1 gene (PQBP1) cause Renpenning syndrome and X-linked mental retardation in another family with microcephaly. Am J Hum Genet. 2004;74(4):777-80. doi:10.1086/383205.

7. Moey C, Hinze SJ, Brueton L, Morton J, McMullan DJ, Kamien B, et al. Xp11.2 micrfoduplication including IQSEC2, TSPYL2 and KDM5C genes in patients with neurodevelopmental disorders. Europ J Hum Genet. 2016;24(3):373-80. doi: 10.1038/ejhg.2015.123. Epub 2015 Jun 10.

8. Waragai M, Lammers $\mathrm{CH}$, Takeuchi S, Imafuku I, Udagawa Y, Kanazawa I, et al. PQBP-1, a novel polyglutamine tract-binding protein, inhibits transcription activation by Brn-2 and affects cell survival. Hum Mol Genet. 1999;8(6):977-87. doi:10.1093/hmg/8.6.977.

9. Okuda T, Hattori H, Takeuchi S, Shimizu J, Ueda H, Palvimo JJ, et al. PQBP-1 transgenic mice show a lateonset motor neuron disease-like phenotype. Hum $\mathrm{Mol}$ Genet. 2003;12(7):711-25. doi:10.1093/hmg/ddg084.

10. Tarpey PS, Smith R, Pleasance E, Whibley A, Edkins S, Hardy C, et al. A systematic, large-scale resequencing screen of X-chromosome coding exons in mental retardation. Nat Genet. 2009;41(5):535-43. doi:10.1038/ ng.367.

11. Arican P, Cavusoglu D, Gencpinar P, Ozyilmaz B, Ozdemir TR, Dundar NO. A de Novo Xp11.23 duplication in a girl with a severe phenotype: Expanding the clinical spectrum. J Pediatr Genet. 2018;7(2):74-77. doi: 10.1055/s-0037-1612598. Epub 2017 Dec 18.

12. dos Santos RC, Barretto OC, Nonoyama K, Castro NH, Ferraz OP, Walter-Moura J, et al. X-linked syndrome: mental retardation, hip luxation, and G6PD variant [Gd(+) Butantan]. Am J Med Genet. 1991;39(2):133136. doi:10.1002/ajmg. 1320390204.

13. Stocco dos Santos RC, Castro NH, Lillia Holmes A, Beçak W, Tackels-Horne D. Stocco dos Santos X-linked mental retardation syndrome: clinical elucidation and localization to Xp11.3-Xq21.3. Am J Med Genet A. 2003;118A(3):255-9. doi:10.1002/ajmg.a.20021. 